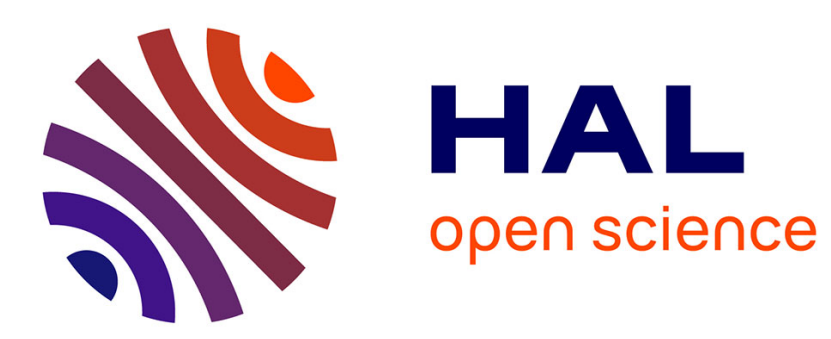

\title{
Channel Assignment Strategies for Optimal Network Capacity of IEEE 802.11s
}

\author{
Carina Teixeira de Oliveira, Fabrice Theoleyre, Andrzej Duda
}

\section{To cite this version:}

Carina Teixeira de Oliveira, Fabrice Theoleyre, Andrzej Duda. Channel Assignment Strategies for Optimal Network Capacity of IEEE 802.11s. PE-WASUN 2012 - 9th ACM International Symposium on Performance Evaluation of Wireless Ad Hoc, Sensor, and Ubiquitous Networks, Oct 2012, Paphos, AA, Cyprus. pp.53-60, 10.1145/2387027.2387037 . hal-00931043

\section{HAL Id: hal-00931043 https://hal.inria.fr/hal-00931043}

Submitted on 2 Jun 2020

HAL is a multi-disciplinary open access archive for the deposit and dissemination of scientific research documents, whether they are published or not. The documents may come from teaching and research institutions in France or abroad, or from public or private research centers.
L'archive ouverte pluridisciplinaire HAL, est destinée au dépôt et à la diffusion de documents scientifiques de niveau recherche, publiés ou non, émanant des établissements d'enseignement et de recherche français ou étrangers, des laboratoires publics ou privés. 


\section{Channel Assignment Strategies for Optimal Network Capacity of IEEE 802.11s}

\author{
Carina T. de Oliveira \\ Grenoble Informatics \\ Laboratory (LIG) \\ University of Grenoble \\ 681, Rue de la Passerelle \\ F38402 Saint Martin d'Hères \\ oliveira@imag.fr
}

\author{
Fabrice Theoleyre \\ CNRS, LSIIT \\ University of Strasbourg \\ Bd Sebastien Brant \\ F67412 Illkirch \\ theoleyre@unistra.fr
}

\author{
Andrzej Duda \\ Grenoble Informatics \\ Laboratory (LIG) \\ University of Grenoble \\ 681, Rue de la Passerelle \\ F38402 Saint Martin d'Hères \\ duda@imag.fr
}

\begin{abstract}
Multi-Channel Multi-Interface (MCMI) Wireless Mesh Networks spread the load across orthogonal channels to improve network capacity. However, due to the limited number of available orthogonal channels in the radio spectrum (3 in IEEE $802.11 \mathrm{~b} / \mathrm{g}$ and 12 in IEEE $802.11 \mathrm{a}$ ), interference and contention are still major factors in such networks. Then, an efficient channel assignment scheme is required to enhance the overall throughput. In this paper, we present three models to evaluate network capacity associated to any channel assignment strategy. These models permit also to extract what we could obtain with an optimal centralized assignment, constituting an upper bound. We present extensive numerical and simulations results, taking into account realistically MAC and routing aspects. In particular, we investigated the impact of the channel assignment strategy onto IEEE 802.11 s, and presented modifications that may improve its performance.
\end{abstract}

\section{Categories and Subject Descriptors}

C.2.1 [Computer-Communication Networks]: [Network Architecture and Design - Wireless Communication]

\section{General Terms}

Experimentation, Performance

\section{Keywords}

multi-channel; multi-interface; channel assignment; capacity; IEEE 802.11s; MILP

\section{INTRODUCTION}

IEEE 802.11-based Wireless Mesh Networks (WMN) have attracted increasing attention in recent years because of their low-cost and ease of deployment. They are composed

Permission to make digital or hard copies of all or part of this work for personal or classroom use is granted without fee provided that copies are not made or distributed for profit or commercial advantage and that copies bear this notice and the full citation on the first page. To copy otherwise, to republish, to post on servers or to redistribute to lists, requires prior specific permission and/or a fee.

Copyright 20XX ACM X-XXXXX-XX-X/XX/XX ...\$10.00. of stationary wireless routers interconnected to form a multihop backbone. Some of them also act as gateways toward the Internet via high-speed wired or wireless links.

Mesh routers are typically equipped with a single IEEE 802.11 wireless network card (an interface) that operates over only a small portion of the available spectrum (a channel). It is well known that such single-interface networks are limited in terms of scale and performance when the node density and the number of hops increases [1], which is mainly due to channel contention and spatial problems such as exposed and hidden nodes.

One way of improving the performance of is to use multiple orthogonal channels (free of inter-channel interference) so that different mesh routers may simultaneously operate on different channels. For instance, IEEE $802.11 \mathrm{~b} / \mathrm{g}$ standards provide 3 orthogonal channels in the $2.4 \mathrm{GHz}$ spectrum, while IEEE 802.11 a provides 12 orthogonal channels in the $5 \mathrm{GHz}$ spectrum. However, the operation on multiple channels has to be carefully designed to avoid deafness: the transmitter must know if the receiver is tuned to the same channel at the same time. Although nodes may have a common reference such as a control channel, a channel hopping order, or periodical rendezvous, they may suffer from low connectivity and even disconnection with frequently channel switches [2].

We can improve the overall capacity of IEEE 802.11-based networks by using multiple interfaces so that different mesh routers may operate on different channels and simultaneously communicate with many neighbors. Such networks are often designated as Multi-Channel Multi-Interface (MCMI) WMN. To take advantage of the increased capacity in such networks, we need to consider several issues: topology formation, radio link scheduling, interference mitigation, and routing [2]. The key issue to be addressed in MCMI WMN and the focus of this paper is channel assignment. We consider this issue in the context of IEEE 802.11s networks.

IEEE 802.11s [3] defines a mesh networking standard for broadcast and unicast packet delivery over a self-configured multi-hop link-layer topology. The current standard does not specify any channel assignment scheme, although such problem is vital to deal with multi-interface nodes. The previous drafts advocated the use of the Common Channel assignment: on every node, channel $C_{1}$ is assigned to interface 1 , channel $C_{2}$ is assigned to interface 2, etc [4], as shown in Fig 1(a). While this strategy is simple to implement and avoids deafness, it leads to a sub-optimal throughput: the 
load is not equally balanced among the different channels [5].

In this paper, we focus on strategies for channel assignment and in particular, on the relation between the assignment and network capacity. We define three Mixed Integer Linear Programming (MILP) formulations to evaluate network capacity in standard IEEE 802.11s networks. We consider the objective function that maximizes throughput while maintaining fairness. Our formulation models routing and bandwidth sharing constraints in presence of interference.

To validate the relevance of such approach, we corroborated numerical results by simulations, which is very seldom done in this research area. In other words, even with realistic conditions and real protocols, do the results obtained numerically keep on holding?

We summarize our contributions as follows.

- We define MILP formulations for the channel assignment problem. They extract the network capacity we may obtain after having assigned one channel to each interface;

- Our formulations are independent on the practical assignment strategies: they permit to compare quantitatively any assignment algorithm;

- We identify ways of improvement for the default IEEE 802.11 strategies;

- We present extensive simulation results to validate the proposed MILP formulations, taking into account aspects neglected in the formulations (realistic MAC layer routing, traffic load). The comparison between different strategies keeps on holding in a more realistic environment.

\section{RELATED WORK}

\subsection{Channel Assignment}

A simple way to assign channels is the Common Channel assignment (on every node, the same set of channels is assigned). Draves et al. [4] follow this approach to propose a routing metric called WCETT for high throughput path selection. As show in Fig. 1(a), a Common Channel solution results in a stable network topology without partitions: two neighbors have always multiple independent links to communicate with each other [5]. While this assignment approach provides suitable stability for routing protocols without path changes and channel switching, it does not efficiently distribute the load among all available channels. Moreover, network capacity degrades with the increase of the network size as more contention and interference may occur.

The Slotted Seeded Channel Hopping (SSCH) [6] adopts synchronized channel hopping sequences such that a pair of nodes shares common timeslots. This dynamic approach has the ability to cover channels with few interfaces, thereby offering the potential to balance the load over different channels and to improve the capacity under heavy load. However, it may alter the network topology: some links are created while others may disappear. These changes can impact upper layers, especially routing protocols.

Wu et al. [7] dedicated one interface to control: it forwards all the reservations (RTS/CTS frames) to negotiate a channel for data exchange. The control channel solves the

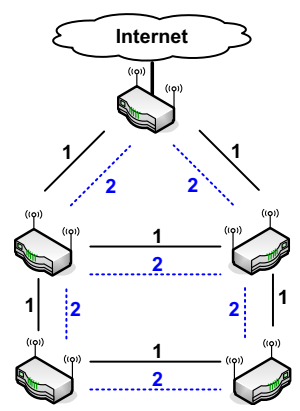

(a) Common Channel Assignment

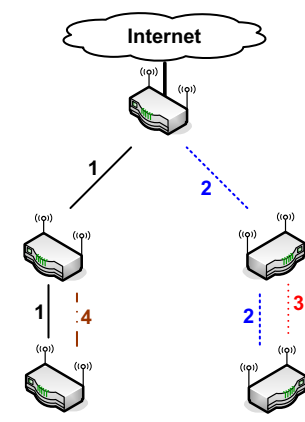

(b) Different Channel Assignment
Figure 1: Two network topologies induced by different channel assignments with 4 orthogonal channels and 2 interfaces.

deafness problem, but introduces a bottleneck interface that may be subject to interference.

In our previous work [5], we have presented a comparison and performance evaluation of different channel assignment strategies addressing connectivity and network topology issues. Our results shown how to improve the MCMI WMN pointing out advantages and limitations of each strategy. In this paper, our goal is to show how to optimize the overall network and seeks the limits of the throughput performance. We believe that the knowledge of bounds is necessary in the design of better algorithms.

\subsection{IEEE 802.11s}

IEEE $802.11 \mathrm{~s}$ [3] has recently standardized the MAC layer in multi-hop wireless mesh networks. It re-uses the CSMACA protocol defined in IEEE 802.11 and slightly adapts it to deal with wireless multi-hop forwarding. IEEE 802.11s proposes to mix reactive and proactive routing to forward packets in multi-hop topologies using the airtime link metric to identify an efficient radio-aware path. This metric estimates the amount of consumed channel resources when transmitting a frame over a particular link. It is defined in terms of transmission errors, data rate, and overhead.

The current standard does not specify a channel assignment scheme (i.e. what channel to use for each interface) although such a scheme is vital in MCMI WMN. While any assignment scheme can be implemented, the previous drafts advocated the use of the Common Channel assignment. However, it leads to many collisions and poor performance in multi-hop topologies $[1,2]$. Fig. 1 illustrates the importance of a channel assignment scheme: a different strategy would assign orthogonal channels to interfaces and avoid collisions between links. Note that the assignment induces the network topology, which can impact on upper layers, especially on routing protocols. Thus, a major challenge in MCMI WMN is how to efficiently assign the available orthogonal channels in order to optimize the network performance. We propose to investigate in this paper the choice of a suitable strategy for IEEE 802.11s.

When multiple interfaces are used, control messages such as Path Request (PREQ) are retransmitted by every interface. As a result, the amount of transmitted control messages exponentially increases with the number of interfaces. This causes severe performance degradation due to 
the broadcast storm problem [8]. We discuss the impact of this misbehavior of the HWMP in Section 5.

\subsection{Wireless Network Capacity}

Gupta et al. [9] pioneered the analysis of capacity in wireless networks. Their main result consists of the observation that capacity decreases when the number of nodes increases. Zemlianov et al. [10] extended the analysis to deal with infrastructure-based wireless networks (some wired links exist). This type of analysis mainly considers asymptotic behavior and does not sufficiently take into account MAC and routing protocols.

Kyasanur and Vaidya [11] studied how network capacity scales with the number of nodes, channels, and interfaces. Franklin et al. [12] considered TDMA-based WMN. They adopted a queuing theory approach, but a comparison of different solutions is not achievable through such a formulation.

Marina et al. [13] presented CLICA, an Integer Linear Programming (ILP) formulation, to obtain a lower bound of the capacity. They derived greedy algorithms to assign channels, however, Kodialam et al. [14] also proposed a MILP formulation. They focused on the TDMA solution and proposed several centralized assignment strategies. Our work rather aims at comparing different approaches and at quantifying the impact of the design choices. We use both an ideal MAC and a TDMA solution to model the MAC layer to tightly estimate the capacity we may obtain with any assignment strategy.

Alicherry et al. [15] have also explored how the routing and MAC layers may be jointly optimized. We rather chose to focus here uniquely on the IEEE 802.11s MAC layer.

\section{NETWORK MODEL}

We consider an IEEE 802.11-based multi-hop multi-channel multi-interface Wireless Mesh Network. We model the network as a graph $G(V, E)$, in which $V$ is the set of vertices and $E$ is the set of edges. Each vertex $u \in V$ corresponds to a wireless node in the network with a known location. There is an edge $(u, v) \in E$ connecting vertex $u$ to $v$ if they are physically located within each other's communication range.

We assume a traffic pattern in which all packets are destined to gateway $G W$ (also denoted as a Mesh Portal). This traffic pattern is common in WMN that provide Internet access to other nodes in the network through the Portal.

We distinguish between two types of interface assignment: static and dynamic. The former assigns each interface to a channel for permanent use [4]. Dynamic assignment allows interfaces to frequently switch from one channel to another [6]. We define a timeslot to be the time spent on a single channel.

Finally, we adopt the concept of conflict graph $G_{C}\left(V_{C}, E_{C}\right)$ to represent interference in $G$ [16] . Each vertex $V_{C}$ represents a link $E \in G$. If two links in $G$ interfere, an edge connecting the two corresponding links in $V_{C}$ is included in $E_{C}$ to represent their conflict. We may use any interfering model that can be expressed as a conflict graph [17].

Let consider the radio topology described in Fig. 2(a). We obtain the conflict-graph in Fig. 2(b). In particular, the links $A B$ and $D E$ are assumed to be sufficiently far to avoid interference. On the contrary, other links are pairwise interfering.

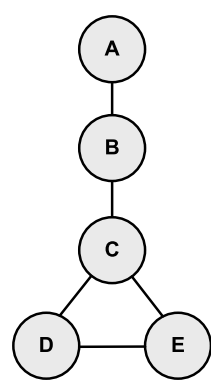

(a) Graph $G$

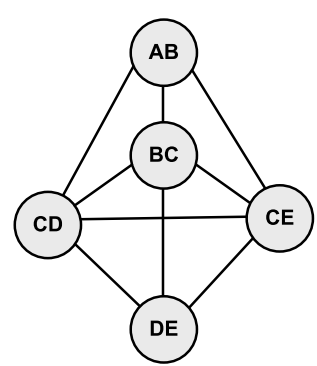

(b) Conflict Graph $G_{C}$
Figure 2: A radio topology and its conflict-graph (two radio links are assumed to be interfering if they are less than two hops far)

Table 1: Notation.

\begin{tabular}{ll}
\hline Symbol & Definition \\
\hline$G W$ & Gateway (Mesh Portal) \\
$N(u)$ & Neighborhood of node $u$ \\
$C h$ & Set of available orthogonal channels \\
$B W$ & Bandwidth of a channel \\
$\mathcal{I}(u)$ & Set of interfaces at node $u$ \\
$T$ & Set of timeslots \\
$n_{s l o t s}$ & Number of timeslots \\
$C l$ & Set of all maximal cliques in conflict graph $G_{c}$ \\
$f(u)$ & Traffic generated by node $u$ \\
$f(u, v)$ & Traffic link $(u, v)$ \\
$f(u, v, c, t)$ & Traffic link $(u, v)$ on channel $c$ and timeslot $t$ \\
$a c t(u, v, c, t)$ & 1 if link $(u, v)$ is active during slot $t$ on channel $c$ \\
$\operatorname{ch}(u, c, t)$ & 1 if node $u$ uses channel $c$ during slot $t$ \\
parent $(u, v)$ & 1 if $v$ is the parent of $u$ in the routing tree \\
\hline
\end{tabular}

\section{MILP FORMULATION}

In this section, we present a novel MILP formulation to evaluate the capacity of channel assignment in IEEE 802.11s. We inject the constraints specific to the assignment into the formulation to obtain the resulting capacity and the optimal assignment. Thus, we can compare different strategies with the optimal upper bound, i.e. what could we obtain with a centralized optimal formulation?

Thus, the optimal upper bound indicates the performance gap to fill with better assignment strategies to maximize the network throughput. Table 1 presents the MILP formulation notation.

\subsection{Objective Function}

We chose an usual objective: we maximize the minimum throughput associated to all the flows. Thus, we guarantee a perfect fairness while maximizing the capacity (max-minflow):

$$
\text { MMFlow }=\operatorname{Max} \quad(\operatorname{Min}(f(u)))
$$

It is subject to the constraints defined below.

\subsection{Constraints}

\subsubsection{Flow Conservation}

Transmitted traffic is equal to the sum of the received 
traffic and the traffic generated by node $u$ itself:

$$
\forall u \in V-\{G W\}, \sum_{v \in N(u)} f(u, v)=\sum_{v \in N(u)} f(v, u)+f(u)
$$

Gateway $G W$ consumes all the flows:

$$
\sum_{u \in N(G W)} f(u, G W)=\sum_{u \in V} f(u)
$$

\subsubsection{Traffic Pattern}

We focus on the convergecast traffic pattern: all the packets are destined to the gateway. However, the model can be directly applied to the bidirectional convergecast traffic: packets are either destined or generated by the gateway. Indeed, constraints 2 and 3 would not change if we consider that $f(u)$ is a bidirectional flow: we would have $\alpha * f(u)$ packets generated by $u$ and $(1-\alpha) * f(u)$ packets transmitted from the gateway to the node $u$. In this scenario, $\alpha$ denotes the ratio between upload and download traffic.

In conclusion, the formulation remains unchanged.

\subsubsection{Multi-Channel Multi-Interface}

Traffic through link $(u, v)$ is the aggregation of the traffic transmitted over all corresponding channels:

$$
\forall(u, v) \in E, \quad f(u, v)=\sum_{c \in \mathcal{C} h, t \in \mathcal{T}} f(u, v, c, t)
$$

A link can forward data only if it is active during a given timeslot on a given channel:

$\forall(u, v) \in E, \forall c \in \mathcal{C} h, \forall t \in \mathcal{T}, f(u, v, c, t) \leq B W \cdot a c t(u, v, c, t)$

We must integrate hardware constraints: the number of interfaces upper bounds the number of channels a node can use. We denote a channel as active for a node if one of its out/ingoing links uses it:

$$
\begin{aligned}
& \forall u \in V, \forall t \in \mathcal{T}, \forall c \in \mathcal{C} h, \\
& \frac{1}{2 *|\mathcal{I}(u)|} \sum_{v \in N(u)}(\operatorname{act}(u, v, c, t)+\operatorname{act}(v, u, c, t)) \leq \\
& \operatorname{ch}(u, c, t) \leq \sum_{v \in N(u)}(\operatorname{act}(u, v, c, t)+\operatorname{act}(v, u, c, t))
\end{aligned}
$$

If a node $u$ has $|\mathcal{I}(u)|$ interfaces, we can then upper bound the number of channels it can use:

$$
\forall u \in V, \forall t \in \mathcal{T}, \quad \sum_{c \in \mathcal{C} h} \operatorname{ch}(u, c, t) \leq|\mathcal{I}(u)|
$$

\subsubsection{Multi/Single flows}

To simplify the formulation, we may forbid multi-flow solutions, i.e. the routing structure forms a tree rooted at gateway $G W$. Each node has in this case a single parent:

$$
\forall u \in V-\{G W\}, \quad \sum_{v \in N(u)} \operatorname{parent}(u, v)=1
$$

Traffic is forwarded only through tree links (in the upload or the download directions):

$\forall(u, v) \in E, f(u, v) \leq B W \cdot|\mathcal{C} h| \cdot(\operatorname{parent}(u, v)+\operatorname{parent}(v, u))$

\subsection{Bandwidth Sharing Constraints}

We have formulated in the previous subsection all hardware (multi-channel/interface) and multi-flow constraints. We have now to model the MAC layer: how the different nodes may share the bandwidth with each other.

We formulate three models to distribute bandwidth of each radio link. We first construct an upper an lower bound modeling an ideal MAC layer: the MAC protocol is able to distribute the radio bandwidth to each contending transmitter with a perfect fairness. This model works with any channel assignment strategy: we just have to fix accordingly the $\operatorname{act}(u, v, c, t)$ variables in the formulation.

We also propose a specific model for a conflict-free assignment, where bandwidth sharing is much simpler to model because no interference arises. If we let the $\operatorname{act}(u, v, c, t)$ unspecified, the MILP resolution will give us the optimal assignment for the given objective.

\subsubsection{Bandwidth Sharing / Upper Bound}

To obtain the upper bound, we forbid two interfering links to be active simultaneously. They may transmit packets during the same timeslot, but not at the same instant, else a collision would occur. Thus, we just have to share the radio bandwidth with fairness in a group of interfering links.

Conflict graph $G_{C}$ provides the right abstraction for formulating such a constraint. We extract all maximal cliques to get the groups of links that interfere pairwisely. A clique is an induced subgraph in $G_{C}$ that is a complete graph. A maximal clique of $G_{C}$ is a complete subgraph that it is not contained in any other complete subgraph. Then, for each maximal clique, all the traffic transmitted by all the radio links of the clique must be inferior to the radio bandwidth:

$$
\forall c l \in \mathcal{C} l, \forall c \in \mathcal{C} h, \forall t \in \mathcal{T}, \sum_{(u, v) \in c l} f(u, v, c, t) \leq B W
$$

We can also remark that two links sharing a node are classified as interfering links. They will share the radio bandwidth: the MAC layer will regulate their transmissions.

We can use the algorithm defined by Bron et al. [18] to extract all maximal cliques in the contention graph. Although the problem is NP-complete, this algorithm leads to an acceptable computing time for small network instances (i.e. a few hundreds of nodes).

We can verify that this constraint is an upper bound: implicitly, we formulate a schedule in a group of radio links that interfere with each other. Although local scheduling is always achievable, we could obtain an infeasible global scheduling. We underestimate here the impact of interference on bandwidth sharing to construct an upper bound.

\subsubsection{Bandwidth Sharing / Lower Bound}

We now construct a feasible solution by creating a globally valid schedule (i.e. a set of radio links that may transmit simultaneously without creating collisions). If we assume that all packets have the same size, we can reference all these sets and give a fraction of the bandwidth to each of the sets. We adopt a TDMA-approach, with $n_{\text {slots }}$ timeslots.

When a schedule is feasible, all the scheduled radio links form a Maximum Independent Set $(\mathcal{M I S})$ in the conflict graph. Indeed, no pair of radio links that are simultaneously activated (are parts of the $\mathcal{M I S}$ ) are neighbors in the conflict graph. Thus, they do not interfere with each other.

We have to reference all the $\mathcal{M I S}$. Then, we assign a 
fraction $q_{i}$ of the bandwidth to each $\mathcal{M I S} i$ : they share the global radio bandwidth. After having referenced all the channels and timeslots, we obtain:

$$
\forall c \in \mathcal{C} h, \forall t \in \mathcal{T}, \sum_{i \in \mathcal{M} \mathcal{I S}} q(i, c, t) \leq \frac{B W}{n_{\text {slots }}}
$$

Finally, each radio link can use at most the sum of the bandwidth assigned to each set it owns:

$$
\begin{aligned}
& \forall(u, v) \in E, \forall c \in \mathcal{C} h, \forall t \in \mathcal{T}, \\
& \qquad(u, v, c, t) \leq \sum_{i \ni(u, v)} q(i, c, t)
\end{aligned}
$$

A radio link will benefit from the sum of bandwidth associated with each $\mathcal{M I S}$ it owns. Combined with inequality 5 , we only count the bandwidth for the channel used by a radio link.

We use the algorithm proposed by Tsukiyama et al. [19] to compute the set of $\mathcal{M I S}$. The number of $\mathcal{M I S}$ can be very large leading to unsolvable problems: it is practically much larger than the number of maximal cliques. This increases the MILP resolution time significantly. Thus, we provide a lower bound by considering only the first $\max _{\mathcal{M I S}}$ sets.

To obtain a feasible solution in which all radio links can be scheduled, each radio link must be present in at least one set of $\mathcal{M I S}$. We proceed in the following way. First, we extract the first $\max _{\mathcal{M I S}}$ groups of radio links $\left(\max _{\mathcal{M I S}}\right.$ is a constant). Then, for each radio link $e$ never present in one group, we create a new set $s=\{e\}$ and greedily insert a list of non-interfering links.

We can verify we construct here a lower bound: by only considering a subset of all $\mathcal{M I S}$, we underestimate the radio spectrum reuse. Some MIS may have not been considered although they would have provided better performance.

\subsubsection{Conflict-free}

We consider separately an assignment strategy where we entirely forbid simultaneous communications of two interfering pairs of nodes. In this case, bandwidth sharing constraints are simpler since a radio link receive the whole radio bandwidth to use: it has no interfering transmitters.

Scheduling has to be found such that no pair of interfering links should be active during the same timeslot (i.e. it is conflict-free). In this case, we reference all cliques in the conflict graph. Therefore, during a given timeslot $t$ only one link per maximal clique is active:

$$
\forall c l \in \mathcal{C} l, \forall c \in \mathcal{C} h, \forall t \in \mathcal{T}, \sum_{(u, v) \in c l} a c t(u, v, c, t) \leq 1
$$

and a radio link can use the full radio bandwidth during $t$ :

$$
\begin{aligned}
& \forall(u, v) \in E, \forall c \in \mathcal{C} h, \forall t \in \mathcal{T}, \\
& \quad f(u, v, c, t) \leq \operatorname{act}(u, v, c, t) \cdot \frac{B W}{n_{\text {slots }}}
\end{aligned}
$$

\section{PERFORMANCE EVALUATION}

In this section, we evaluate the capacity of IEEE $802.11 \mathrm{~s}$ through numerical analysis and simulation. We focus on the most interesting results that can guide the design of an efficient protocol in MCMI WMN. We present the results with a confidence level of $95 \%$.

\section{$5.1 \quad$ Numerical Results}

We first analyze the results of the MILP formulation for the following strategies. We use the notation Channel Assignment Strategy / MILP bandwidth sharing constraints to designate each approach:

- Common Channel / Bandwidth Sharing UP and LOW (upper and lower bounds): channel $C_{k}$ is assigned to interface $k$ [4]. The MILP upper and lower bounds (Sections 4.3.1 \& 4.3.2) extract the associated capacity since some interfering interfaces may use the same channel;

- MMFlow / Bandwidth Sharing UP and LOW (upper and lower bounds): we let the MILP decide by itself the channel assignment while maximizing the network capacity (Eq. 1). The MILP upper and lower bounds (Sections 4.3.1 \& 4.3.2) formulate bandwidth sharing constraints between possibly interfering interfaces;

- MMFlow / Conflict-free (Section 4.3.3): we use the MILP formulation which forbids interfering links to be active in the same timeslot. The MILP assigns during the resolution one channel per interface and timeslot.

A custom simulator ${ }^{1}$ generates Unit Disk Graphs and we consider the interference range is twice the radio range [17].

Nodes are placed randomly on a disk while maintaining an average degree of 7 (number of neighbors). We use 10 channels in the $5 \mathrm{GHz}$ band to stress the channel assignment (more IEEE 802.11a channels means a larger capacity). Numerical simulations measure the MILP objective function MMFlow (cf. Eq. 1).

\subsubsection{Impact of the number of interfaces}

Figure 3(a) shows the throughput of each flow (obtained through the objective function in Eq. 1) as the number of interfaces increases. The capacity almost linearly increases with the number of interfaces for all the strategies. Kyasanur et al. [11] obtained similar results with an asymptotic approach. Thus, even if we take into account protocol details, the result keeps on holding: using a larger number of interfaces is always beneficial.

We can also remark that Common Channel assignment is a sub-optimal strategy. Although it is simple to implement, it negatively impacts capacity. Finally, we may also note that MMFlow/Conflict-free and MMFlow/BwSharing offer similar capacity: a simple conflict-free assignment leads to the same throughput as with an ideal MAC layer. As this fact may significantly simplify implementation, it is a promising way to the design of efficient IEEE 802.11 s networks.

\subsubsection{Dynamic interfaces}

Furthermore, we study the impact of the interface assignment strategy (static versus dynamic) on the capacity. For MMFlow/BwSharing, we have modified the number of slots: 1 slot means that interfaces are static, while a larger number of slots increases the number of channel switching (Fig. 3(b)). Surprisingly, we remark that network capacity does not depend on the number of slots, i.e. number of

\footnotetext{
${ }^{1}$ The opensource simulator is available at https://forge. imag.fr/projects/graphsim.
} 


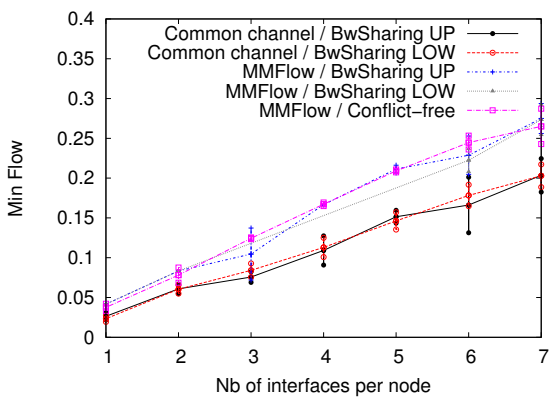

(a) 4 slots

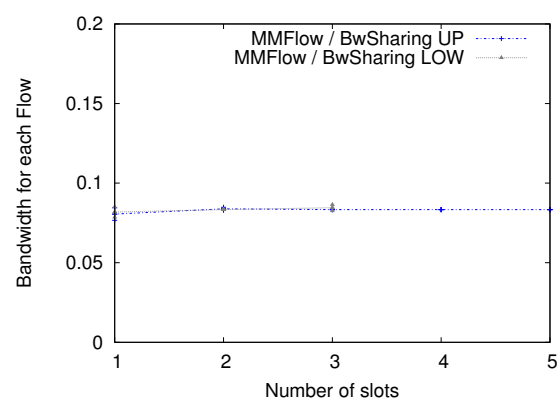

(b) 2 interfaces

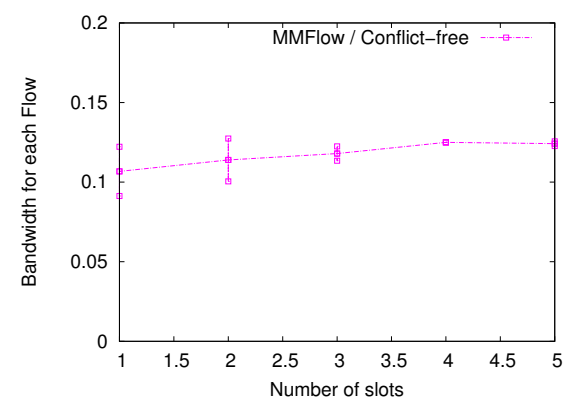

(c) 2 interfaces

Figure 3: Numerical results (25 nodes, 10 channels) - Bandwidth for each flow is obtained through the MILP (cf. Eq. 1), "Bwsharing "stands for "Bandwidth sharing", "UP" and "LOW" stand respectively for Upper and Lower Bounds

channel switching. Thus, static interfaces permit to obtain optimal capacity while limiting implementation complexity.

\subsubsection{Conflict-Free scheduling complexity}

Finally, we focus on the MMFlow/Conflict-free scheduling approach (Fig. 3(c)). To stress IEEE 802.11, we set-up one interface per node to forward packets. Surprisingly, capacity is almost independent of the number of slots. In other words, static channel assignment is sufficient to attain near optimal capacity.

Thus, IEEE 802.11s can achieve optimal performance, if all interfaces use a static channel during a given timeslot, and a distributed algorithm implements a conflict-free channel assignment (i.e. during a given timeslot, no pair of interfering radio links use the same channel).

\subsection{Simulation Results}

We have validated the MILP formulation by simulations in NS-3.12 using the IBM ILOG CPLEX Optimizer to solve linear programming presented in Section 4 . We have developed extensions to support the interaction between NS3 and CPLEX, as well as to support different channel assignments. We depict this interaction in Fig. 5: a box denotes an action and an arrow represents variables passing between actions. The first step is to define the topology in NS-3. We use a single-interface network tuned to a common channel to run a neighbor discovery process (without data traffic) during 30 seconds and obtain the network topology. This topology is passed to CPLEX along with a list of parameters such as the number of channels and interfaces, the number of timeslots, and the bandwidth sharing mode (MMFlow/BwSharing UP, MMFlow/BwSharing LOW, or MMFlow/Conflict-free). Then, CPLEX solves the MILP problem and returns to NS-3 the list of channels to be assigned to each interface of each node. Finally, we evaluate the channel assignment reported by CPLEX through NS-3 simulations in a MCMI WMN.

Simulations validated the assumptions we used in our MILP model: even if the PHY and MAC layers are more complex, the different assignment strategies keep on achieving different network capacity.

We evaluate the performance with 49 nodes arranged in a grid of 7 by 7 . Each node has at most 4 neighbors in the grid. Nodes are equipped with IEEE 802.11a wireless interfaces and therefore 12 orthogonal channels are available. To represent a typical WMN traffic pattern, flows are originated from the mesh routers to the gateway. Each data point in the graphs is computed as being a result of 40 different simulations. For each simulation run, a node is randomly designated as the gateway to receive the Poisson traffic generated by all other mesh routers. A new flow is started every 1 second. Thus, the variations in the obtained results mainly occur due to the randomness of the topology caused by the gateway position and flow initialization. We have considered a packet size of 512 bytes. The total simulation time is 100 seconds.

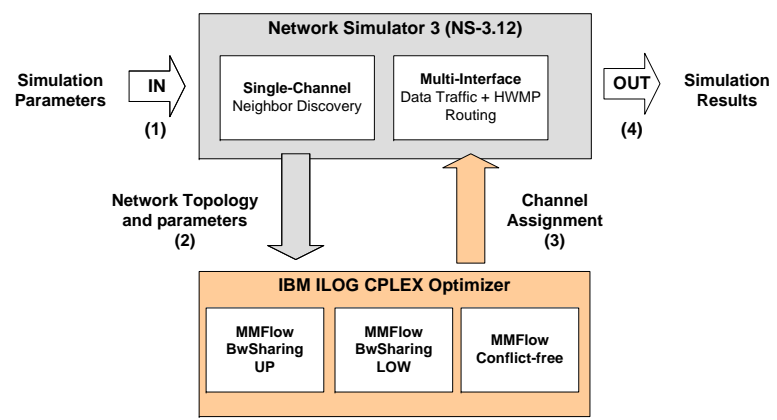

Figure 5: A diagram that depicts the interaction between NS-3.12 and CPLEX.

\subsubsection{Network capacity}

We first investigate the impact of an increasing data traffic (Fig. 4). We observe that all MILP solutions present better delivery rate (Fig. 4(a)) and aggregated throughput (Fig. 4(b)) than the Common Channel assignment approach regardless of the traffic load. Moreover, as the data rate increases, the performance gap between the proposed MILP solutions and the Common Channel assignment also increases. As expected, the MMFlow/Conflict-free solution presents the highest throughput: by limiting interference and collisions, it improves the performance. Also, note that the performance of MMFlow/BwSharing UP is lower than MMFlow/BwSharing LOW. Under-estimating interference leads practically to a less efficient channel assignment.

We then studied the routing behavior measuring in particular the number of transmitted Path ERRors (PERR). The PERR is used for announcing unreachable destinations. As shown in Fig. 4(d), too many packet transmissions increase the error rate. We highlight that the high error rate increases the path cost and leads to frequent path changes. This path instability in HWMP leads to the degradation of the delivery 


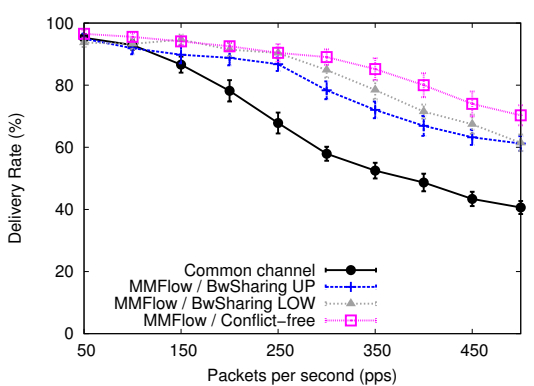

(a) Delivery Rate.

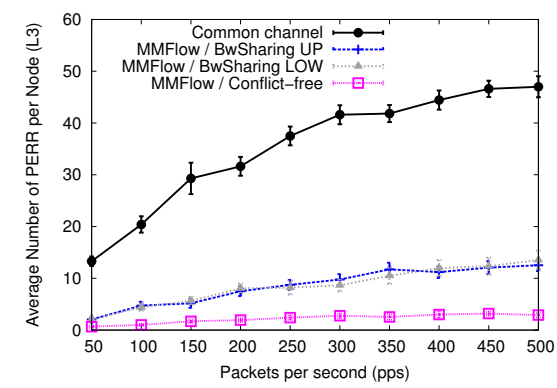

(d) Path Error (PERR) L3.

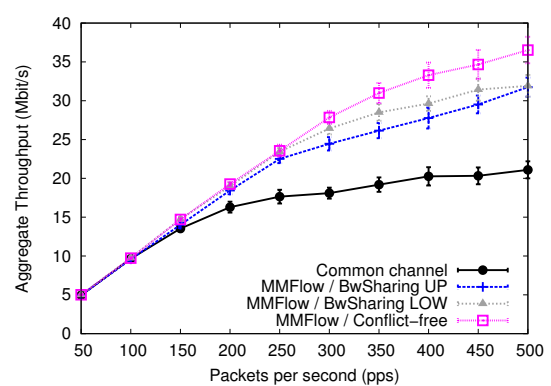

(b) Aggregate Throughput.

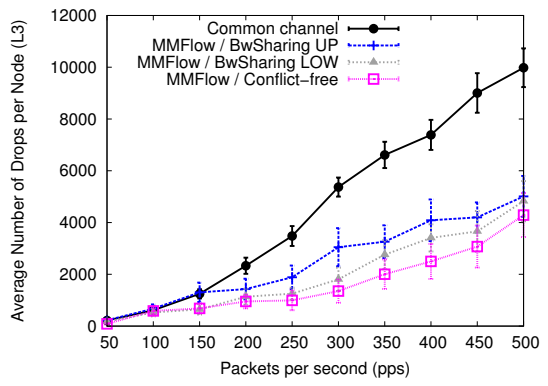

(e) Drop Network Layer.

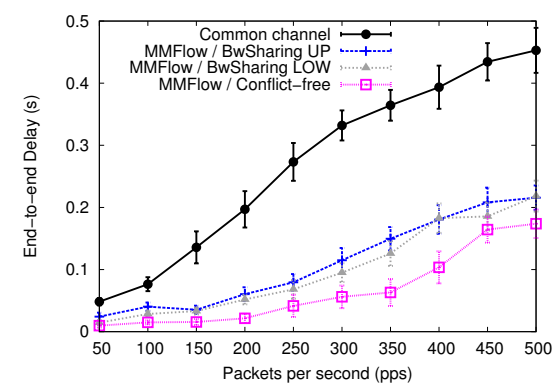

(c) End-to-end Delay.

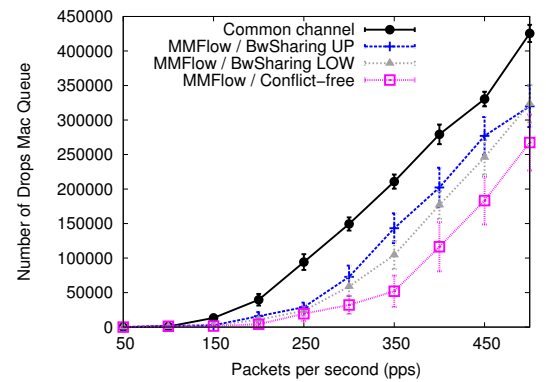

(f) Drop MAC Queue.

Figure 4: Simulation results. Impact of data traffic (3 interfaces, 12 channels).

rate (Fig. 4(a)) and increases delay (Fig. 4(c)), especially for the Common Channel assignment, because it operates only over a small portion of the available spectrum. Thus, it does not efficiently distribute the load among all available channels as in the proposed MILP formulation. Therefore, more contention and interference may occur on pre-defined channels in the Common Channel assignment.

In addition, the Common Channel assignment suffers from severe performance degradation due to the broadcast storm problem: HWMP broadcast many control packets such as the Path Request (PREQ) on each of its interfaces, which results in serious overhead, collisions, and contention. Indeed, two neighbors in the Common Channel assignment always have multiple independent links to communicate with each other. Besides, the amount of transmitted and received control packets in the Common Channel assignment is much higher than that of MILP approaches. Therefore, while a considerable part of transmission opportunities is used by the Common Channel assignment to send control packets, these opportunities are used by the MILP strategies to send data packets. In particular, the MMFlow/Conflict-free case performs much better than the Common Channel assignment especially under high data traffic, because it computes a schedule for radio links so that collisions and interference are avoided.

In IEEE 802.11 s, a node may have several network interfaces with different MAC addresses. Thus, the standard defines a network layer buffer for packets waiting for routes and eventual forwarding to the next hop. Fig. 4(e) shows the number of packet drops at the network layer (i.e. route was not found), while Fig. 4(f) presents the number of frame drops at the MAC layer. We can notice that in multi-hop forwarding, the same buffer is used for both the traffic forwarded from other interfaces and traffic originated by the node itself. Thus, the nodes close to the gateway will quickly undergo buffer overflow, an effect known as the queue con- tention problem, which creates an unfair situation for the traffic originated by the nodes close to the gateway. The fall of the curves when the data traffic increases in Fig. 4(a) comes in part from this unfair behavior. We believe that the basic means to address this issue is to give channel assignment priority to links closer to the gateway based on the number of available channels and interfaces per node.

\subsubsection{Impact of the number of interfaces}

Next, we study the impact of the number of interfaces on the capacity (Fig. 6). For all MILP solutions, delivery rate and aggregate throughput slightly increase and the end-toend delay slightly decreases with the number of interfaces. This limitation of the increase of capacity mainly occurs because of the queue contention problem and the retransmission of control packets by every interface. For the Common Channel assignment, a greater number of interfaces increases the amount of transmitted control messages. For example, PREQs are retransmitted by every interface.

It is clear that the default IEEE 802.11s routing protocol (HWMP) is not as good for MCMI networks. Therefore, the improvement in fairness and the reduction of overhead without affecting routing protocol performance are two challenging issues to achieve better capacity in MCMI IEEE 802.11s.

\section{CONCLUSIONS AND FUTURE WORK}

In this paper, we have presented MILP formulations to evaluate network capacity in multi-channel multi-interface wireless mesh networks. They allow us to quantitatively study various design choices for IEEE 802.11s and in particular, to show that static assignment is beneficial in convergecast mesh networks.

We have also run extensive simulations that take into account aspects neglected in the MILP formulations (realistic MAC layer, routing, traffic load). The results confirm 


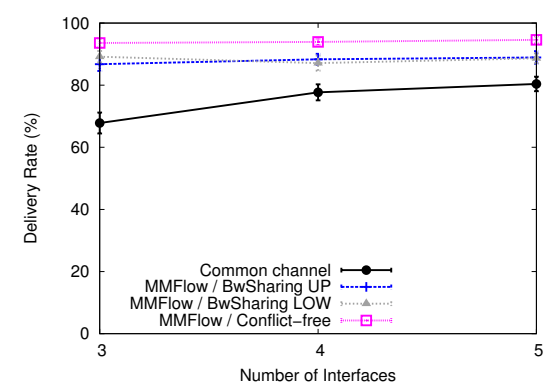

(a) Delivery Rate.

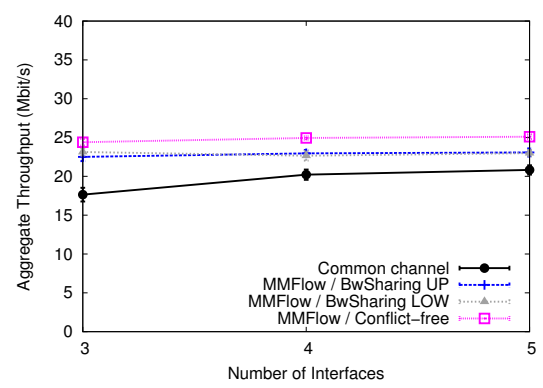

(b) Aggregate Throughput.

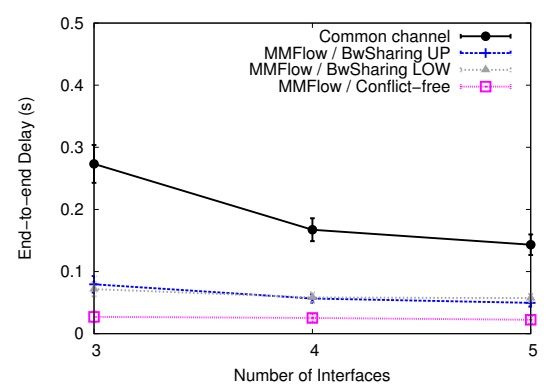

(c) End-to-end Delay.

Figure 6: Simulation results. Impact of the number of interfaces (12 channels, $250 \mathrm{pps})$.

the validity of the conclusions from the analysis based on the MILP formulations. The Common Channel assignment strategy is clearly sub-optimal and a large performance gap has still to be filled. One direction for improvement is the use of conflict-free solutions that present the highest throughput by limiting interference and collisions.

In the future, we plan to propose modifications to IEEE 802.11 s to enable conflict-free assignment. We aim to investigate how to cope with the queue contention problem by using different queuing schemes and forms of fairness.

\section{REFERENCES}

[1] C. Chaudet, D. Dhoutaut, and I. G. Lassous. Performance Issues with IEEE 802.11 in Ad Hoc Networking. IEEE Communications Magazine, 43(7), 2005.

[2] W. Si, S. Selvakennedy, and A. Y. Zomaya. An Overview of Channel Assignment Methods for Multi-Radio Multi-Channel Wireless Mesh Networks. Journal of Parallel and Distributed Computing, 70(5):505-524, 2010.

[3] 802.11s, IEEE Standard for Information Technology Telecommunications and Information Exchange Between Systems - Local and Metropolitan Area Networks - Specific Requirements - Part 11: Wireless LAN Medium Access Control (MAC) and Physical Layer (PHY) Specifications - Amendment 10: Mesh Networking, IEEE standard, 2011.

[4] R. Draves, J. Padhye, and B. Zill. Routing in Multi-Radio, Multi-Hop Wireless Mesh Networks. In Proc. ACM MobiCom, 2004.

[5] C. T. De Oliveira, F. Theoleyre, and A. Duda. Connectivity in Multi-Channel Multi-Interface Wireless Mesh Networks. In Proc. IEEE IWCMC, 2011.

[6] P. Bahl, R. Chandra, and J. Dunagan. SSCH: Slotted Seeded Channel Hopping for Capacity Improvement in IEEE 802.11 Ad-Hoc Wireless Networks. In Proc. ACM MobiCom, 2004.

[7] S.-L. Wu, Y.-C. Tseng, C.-Y. Lin, and J.-P. Sheu. A Multi-Channel MAC Protocol with Power Control for Multi-Hop Mobile Ad Hoc Networks. The Computer Journal, 45(1):101-110, 2002.

[8] S.-Y. Ni, Y.-C. Tseng, Y.-S. Chen, and J.-P. Sheu.
The Broadcast Storm Problem in a Mobile Ad Hoc Network. In Proc. ACM MobiCom, 1999.

[9] P. Gupta and P. R. Kumar. The Capacity of Wireless Networks. IEEE Transactions on Information Theory, 46(2):388-404, 2000.

[10] A. Zemlianov and G. De Veciana. Capacity of Ad Hoc Wireless Networks With Infrastructure Support. IEEE Journal on Selected Areas in Comunications, 23:657-667, 2005.

[11] P. Kyasanur and N. H. Vaidya. Capacity of Multi-Channel Wireless Networks: Impact of Number of Channels and Interfaces. In Proc. ACM MobiCom, 2005.

[12] A. A. Franklin, V. Bukkapatanam, and C. S. R. Murthy. Impact of Multiple Channels and Radios on the Performance of a TDMA Based Wireless Mesh Network. Computer Networks, 53:2208-2224, 2009.

[13] M. K. Marina, S. R. Das, and P. Subramanian. A Topology Control Approach for Utilizing Multiple Channels in Multi-Radio Wireless Mesh Networks. Computer Networks, 54:241-256, 2010.

[14] M. Kodialam and T. Nandagopal. Characterizing the Capacity Region in Multi-Radio Multi-Channel Wireless Mesh Networks. In ACM MobiCom, 2005.

[15] M. Alicherry, R. Bhatia, and L. E. Li. Joint Channel Assignment and Routing for Throughput Optimization in Multiradio Wireless Mesh Networks. IEEE Journal on Selected Areas in Communications, 24(11):1960 -1971, 2006.

[16] K. Jain, J. Padhye, V. Padmanabhan, and L. Qiu. Impact of Interference on Multi-Hop Wireless Network Performance. In Proc. ACM MobiCom, 2003.

[17] A. Iyer, C. Rosenberg, and A. Karnik. What is the Right Model for Wireless Channel Interference? IEEE Transactions on Wireless Communications, 8(5):2662-2671, 2009.

[18] C. Bron and J. Kerbosch. Algorithm 457: Finding All Cliques of an Undirected Graph. Communications of the ACM, 16:575-577, 1973.

[19] S. Tsukiyama, M. Ide, H. Ariyoshi, and I. Shirakawa. A New Algorithm for Generating All the Maximal Independent Sets. SIAM Journal on Computing, 6:505-517, 1977. 